\title{
Article
}

\section{Transmission and Emission of Solar Energetic Particles in Semi-transparent Shocks.}

Kocharov, Leon, Laitinen, Timo Lauri Mikael, Usoskin, Ilya and Vainio, Rami

Available at https://clok.uclan.ac.uk/10590/

Kocharov, Leon, Laitinen, Timo Lauri Mikael orcid iconORCID: 0000-00027719-7783, Usoskin, llya and Vainio, Rami (2014) Transmission and Emission of Solar Energetic Particles in Semi-transparent Shocks. Astrophysical Journal Letters, 787 (2). L21.

It is advisable to refer to the publisher's version if you intend to cite from the work. http://dx.doi.org/10.1088/2041-8205/787/2/L21

For more information about UCLan's research in this area go to http://www.uclan.ac.uk/researchgroups/ and search for <name of research Group>.

For information about Research generally at UCLan please go to http://www.uclan.ac.uk/research/

All outputs in CLoK are protected by Intellectual Property Rights law, including Copyright law. Copyright, IPR and Moral Rights for the works on this site are retained by the individual authors and/or other copyright owners. Terms and conditions for use of this material are defined in the policies page.

\section{CLoK}

Central Lancashire online Knowledge www.clok.uclan.ac.uk

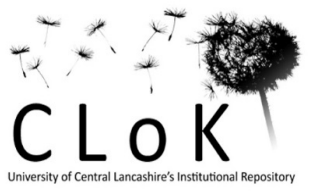




\title{
TRANSMISSION AND EMISSION OF SOLAR ENERGETIC PARTICLES IN SEMI-TRANSPARENT SHOCKS
}

\author{
Leon Kocharov $^{1}$, Timo Laitinen ${ }^{2}$, Ilya Usoskin ${ }^{1,3}$, and Rami Vainio ${ }^{4,5}$ \\ ${ }^{1}$ Sodankylä Geophysical Observatory (Oulu Unit), University of Oulu, P.O. Box 3000, FI-90014 Oulu, Finland \\ ${ }^{2}$ Jeremiah Horrocks Institute, University of Central Lancashire, Preston PR1 2HE, UK \\ ${ }^{3}$ Department of Physics, University of Oulu, FI-90014 Oulu, Finland \\ ${ }^{4}$ Department of Physics and Astronomy, University of Turku, FI-20014 Turku, Finland \\ ${ }^{5}$ Department of Physics, University of Helsinki, FI-00014 Helsinki, Finland \\ Received 2014 March 13; accepted 2014 April 26; published 2014 May 13
}

\begin{abstract}
While major solar energetic particle (SEP) events are associated with coronal mass ejection (CME)-driven shocks in solar wind, accurate SEP measurements reveal that more than one component of energetic ions exist in the beginning of the events. Solar electromagnetic emissions, including nuclear gamma-rays, suggest that high-energy ions could also be accelerated by coronal shocks, and some of those particles could contribute to SEPs in interplanetary space. However, the CME-driven shock in solar wind is thought to shield any particle source beneath the shock because of the strong scattering required for the diffusive shock acceleration. In this Letter, we consider a shock model that allows energetic particles from the possible behind-shock source to appear in front of the shock simultaneously with SEPs accelerated by the shock itself. We model the energetic particle transport in directions parallel and perpendicular to the magnetic field in a spherical shock expanding through the highly turbulent magnetic sector with an embedded quiet magnetic tube, which makes the shock semi-transparent for energetic particles. The model energy spectra and time profiles of energetic ions escaping far upstream of the shock are similar to the profiles observed during the first hour of some gradual SEP events.
\end{abstract}

Key words: acceleration of particles - shock waves - Sun: corona - Sun: coronal mass ejections (CMEs) Sun: flares - Sun: particle emission

Online-only material: color figures

\section{INTRODUCTION}

The appearance of solar energetic particle (SEP) events is highly variable depending on the particle energy range, from $\sim 1-10 \mathrm{MeV}$ to $\sim 0.1-10 \mathrm{GeV}$, the event class, impulsive or gradual, and other solar and interplanetary factors (e.g., Cliver \& Ling 2007; Gopalswamy et al. 2012, and references therein). Even selecting only major (gradual) SEP events, the observed time-intensity profiles and particle abundances indicate that more than one SEP component will emerge during the first hour of the event (e.g., Figure 1 by Kocharov et al. 2012). Origin of ground level events (GLEs) that occupy the high-energy end of gradual SEP events is still obscure (Nitta et al. 2012). A simple scenario with a single SEP source may not be sufficient to explain the variety of the particle components and evolutions observed in major events.

Models for the main phase acceleration of gradual events consider particle acceleration in the parallel shock waves on open magnetic field lines of solar wind (Lee \& Ryan 1986; Lee 2005; Vainio \& Laitinen 2007; Ng \& Reames 2008). However, shock acceleration can be even more efficient in the magnetic environment of solar corona (e.g., Kocharov et al. 2012), and the prompt production of high-energy protons may be associated with global coronal waves (Kocharov et al. 1994; Torsti et al. 1999). If the main SEP peak is produced by the shock driven by a coronal mass ejection (CME) in the solar wind, the additional, early components of SEPs should be attributed to coronal shocks or other CME-liftoff processes near the Sun.

Diffusive shock acceleration can operate in different astrophysical objects if the particle scattering is sufficiently strong, producing a power-law energy spectrum of accelerated particles near and downstream of the shock (Krymsky 1977; Axfordet al.
1977; Bell 1978; Blandford \& Ostriker 1978). The strong scattering in the vicinity of the shock front is expected to shield any particle source situated behind the shock. However, the original model of diffusive shock acceleration undergoes different modifications (e.g., recent Kocharov et al. 2013), which may change the understanding of particle transport from the sources situated behind the CME-bow shock.

In this Letter, we consider energetic particle transport in the realistic semi-transparent shock model that for the first time reproduces the simultaneous emission into the interplanetary space of particles accelerated at the shock front and particles from possible coronal sources behind the shock. The model is motivated by the broadband observations on the Solar and Heliospheric Observatory ( $\mathrm{SOHO}$ ) spacecraft and neutron monitors on the ground.

\section{OBSERVATIONAL AND THEORETICAL MOTIVATION}

The SEP profiles observed at the Earth's orbit are affected by the particle scattering by the turbulence in the solar wind. However, the solar wind turbulence level is highly variable and the mean free path of $\gtrsim 10 \mathrm{MeV}$ protons at the Earth's orbit may vary from $\sim 0.1 \mathrm{AU}$ to $\sim 10 \mathrm{AU}$. For instance, a detailed analysis of the GLE and SEP event of 1998 May 2 (Kocharov et al. 2007b, 2007a) has revealed several jumps in the particle scattering conditions as the particle detector moved from one magnetic flux tube to another. Onset of the SEP event was observed in an extremely quiet magnetic tube with the fitted mean free path value of at least $10 \mathrm{AU}$, while much smaller values were met in neighboring magnetic tubes later in the event (Torsti et al. 2004). The almost scatter-free particle transport in the first phase of the 1998 May 2 event can 


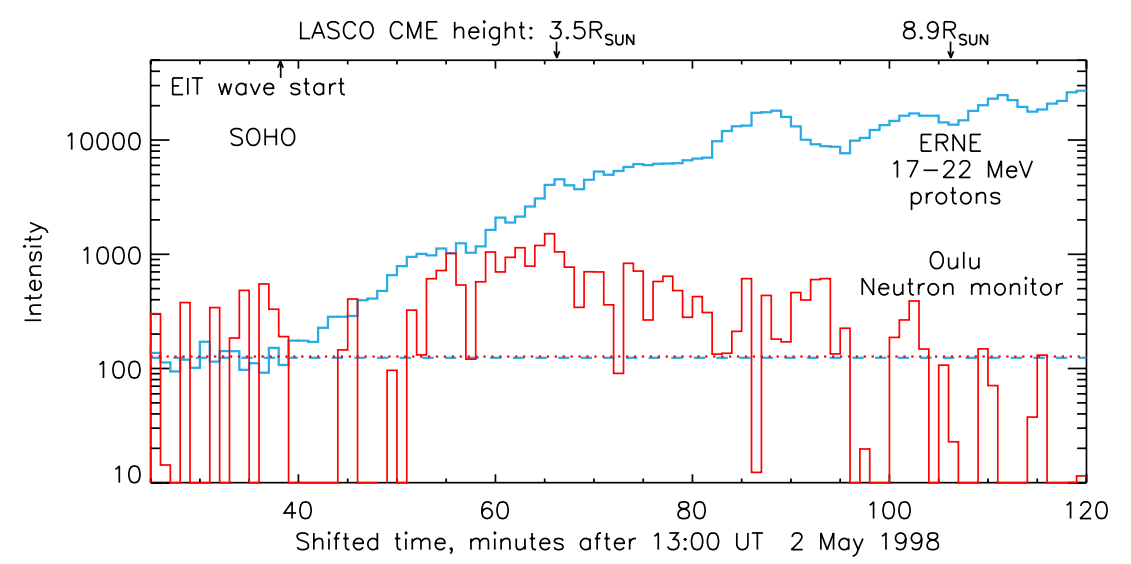

Figure 1. Major SEP event and GLE of 1998 May 2 as observed in space with the Energetic and Relativistic Nuclei and Electron (ERNE) instrument on the SOHO spacecraft and on the ground with the Oulu neutron monitor (both profiles are shifted in time and renormalized for the sake of mutual comparison and comparison with solar electromagnetic emissions). Particle time profiles are shifted back by 39 minutes for the ERNE channel and by 3 minutes for the neutron monitor, which is the corresponding proton flight time from the Sun to the Earth, but 8 minutes. The neutron monitor background level has been reduced to that of ERNE by subtracting $99 \%$ of the 30 minute average galactic background (dotted red and dashed blue lines are for the equalized backgrounds). Two profiles are easily comparable after the equalization of the backgrounds, near equalization of the signals, and compensation for the velocity dispersion. It is seen that the history of the $\gtrsim 400 \mathrm{MeV}$ proton emission that is responsible for the ground level event is distinct from the history of deka-MeV protons. Oulu neutron monitor data are available at http://cosmicrays.oulu.fi.

(A color version of this figure is available in the online journal.)

justify the time-shifting method for the analysis of the particle emission history at the Sun. In this method the time-intensity profiles of high-energy particles observed at $1 \mathrm{AU}$ are simply shifted in time back to the Sun by subtracting the particle flight time along the interplanetary magnetic field line. Then the light flight time from Sun to Earth is added for comparison with solar electromagnetic emissions observed at the Earth's orbit. Figure 1 shows the shifted time profile of deka-MeV protons observed in space and the shifted profile of the neutron monitor count rate caused by $>400 \mathrm{MeV}$ nucleon $^{-1}$ solar protons and helium. Previous detailed analysis of the $14-140 \mathrm{MeV}$ proton profiles in nine energy channels led to the conclusion that there were two nearly simultaneous components of high-energy protons emitted from the Sun during the first hour of the event: the lower energy component, $<50 \mathrm{MeV}$ nucleon $^{-1}$, and the higher energy component, $>50 \mathrm{MeV}$ nucleon $^{-1}$. Those components were different in terms of the onset behavior, energy spectrum, and helium-to-proton abundance ratio, and hence require two different sources, e.g., a coronal shock in addition to the CMEbow shock in the solar wind.

In the strongest events, solar protons can be accelerated to energies well above $300 \mathrm{MeV}$. The presence of such high-energy ions at the Sun is evident from the high-energy gamma-ray emission observed, for instance, in the solar eruption of 1990 May 24 (Kocharov et al. 1994, 1996, and references therein). On the other hand, the high-energy ions escaping into the interplanetary medium can be detected by particle instruments in space and by neutron monitors on the ground. Integrated analysis of high-energy protons, secondary gamma-rays, and neutrons, along with the neutron-decay protons, in the 1990 May 24 event support the idea that a delayed component of highenergy protons interacting at the Sun was similar to the prompt component of accelerated protons emitted into the interplanetary space. The high-energy protons of that GLE and the high-energy protons producing gamma-rays and neutrons at the Sun are likely to originate from the common coronal source.

Recently, we modeled the proton acceleration and transport in the planar shock propagating through the intermittent solar wind that comprises both the turbulent magnetic tubes and the quiet magnetic tubes (Kocharov et al. 2013). Particles are accelerated in the highly turbulent tubes and escape upstream of the shock via the quiet tubes. However, such quiet tubes could simultaneously allow particles from additional sources behind the shock to escape together with the shock-accelerated particles. Thus, we now introduce an additional source of high-energy particles behind the shock and consider a more realistic, spherical shock, which affects both the shock-accelerated component and the added component that will be modified during its transmission through the shock. The cross-field diffusion is included and will allow SEPs from both sources to appear on the same line of the interplanetary magnetic field. Similar to the previous modeling, we assume that the high-energy particle density is sufficiently small to neglect an effect of the particles on their resonant waves.

\section{NUMERICAL MODEL}

We model a spherical shock in the radial magnetic field $\boldsymbol{B}=$ $B_{\odot}\left(R_{\odot} / r\right)^{2} \boldsymbol{r} / r$. Particle injection and transport parameters are assumed to have symmetry around the polar axis, $z$, and hence the cross-field diffusion can only be traced in the $\theta$ direction (Figure 2). The standard diffusion-convection equation of cosmic ray transport for the volumetric number density, $F$, (e.g., Toptygin 1985) has been rewritten for the number of particles per unit of magnetic tube length, $N(\boldsymbol{r}, E, t)=F(\boldsymbol{r}, E, t) \Phi / B(\boldsymbol{r}, t)$, where $F(\boldsymbol{r}, E, t)$ is the number of particles of energy, $E$, per unit of volume, $B$ is the magnetic field, and $\Phi$ is a constant with dimensionality of magnetic flux. In the perpendicular diffusion term,

$$
\left(\frac{\partial N}{\partial t}\right)_{\perp}=\frac{1}{r^{2} \sin \theta} \frac{\partial}{\partial \theta} D_{\perp} \sin \theta \frac{\partial N}{\partial \theta},
$$

we replace the polar angle, $\theta$, with the virtual coordinate, $Y$, that is defined as

$$
Y=(1-\cos \theta) R_{o}
$$




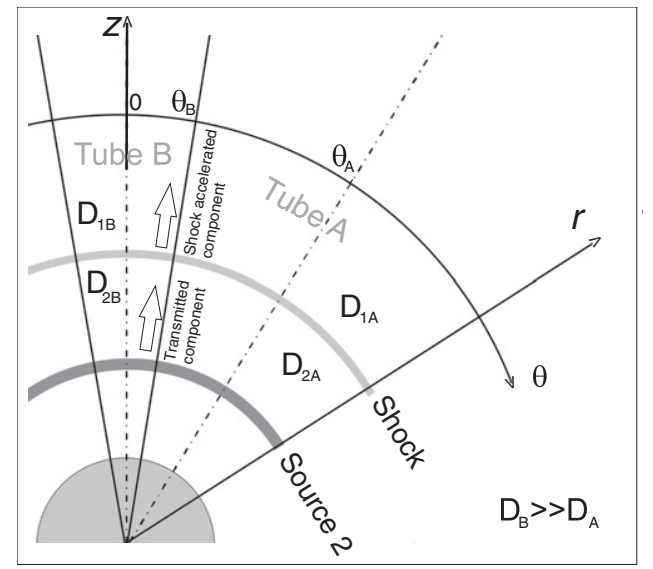

Figure 2. Particle acceleration and transport model. Low-energy seed particles of upstream plasma are promptly accelerated by the shock in the highly turbulent sector (tube A) and, after cross-field transport, can escape far upstream of the shock through the quiet tube B. An additional SEP source is situated at a fixed position well behind the shock (Source 2). High-energy particles from this source can transverse the shock and escape along tube B. Some of them are reaccelerated at the shock in tube A. Actual size of the simulation box cone is $\theta_{\mathrm{A}}=5.73 ; \theta_{\mathrm{B}}=\theta_{\mathrm{A}} / 2$. The SEP acceleration region in real gradual events is much wider and may comprise a number of quiet and turbulent tubes.

where $R_{o}$ is a normalization length. Finally, the field-alighted diffusion-convection equation is of the form

$$
\begin{aligned}
\frac{\partial N}{\partial t}= & \frac{\partial}{\partial r} D_{\|} \frac{\partial N}{\partial r}-\frac{\partial}{\partial r}\left(u+\frac{2 D_{\|}}{r}\right) N \\
& +\frac{\partial}{\partial Y} D_{\perp}\left(\frac{R_{o}}{r}\right)^{2}\left(2-\frac{Y}{R_{o}}\right) \frac{Y}{R_{o}} \frac{\partial N}{\partial Y} \\
& +\frac{1}{3} \frac{\partial}{\partial E}(a E N) \nabla \cdot \boldsymbol{u},
\end{aligned}
$$

where $a=d \ln E / d \ln p$; and $E$ and $p$ are the particle's kinetic energy and momentum, respectively. This equation is solved with stochastic simulations of the random walk and advection of "Monte Carlo particles."

The region under consideration comprises the highly turbulent cone A (tube A) with embedded quiet cone B (tube B; Figure 2). We adopt $R_{o}=200 R_{\odot}$, which for the angular size of the simulation box $\theta_{\mathrm{A}}=0.1 \mathrm{rad}$ gives the corresponding size $Y_{\mathrm{A}} \equiv Y\left(\theta_{\mathrm{A}}\right)=R_{\odot}$, and for the tube B boundary $\theta_{\mathrm{B}}=0.05 \mathrm{rad}$ we have $Y_{\mathrm{B}}=\left(\theta_{\mathrm{B}} / \theta_{\mathrm{A}}\right)^{2} Y_{\mathrm{A}}=0.25 R_{\odot}$. The simulation box in the $r-Y$ plane is rectangular, while the spherical geometry of the system comes to the coordinate dependence of the $Y$-diffusion coefficient, $D_{Y}(r, Y) \approx 2 D_{\perp} R_{o} Y / r^{2}$. Similar to Kocharov et al. (2013), we adopt the energy-dependent parallel diffusion coefficient as $D_{\|}(E) \propto E^{0.75}$ and the perpendicular diffusion coefficient $D_{\perp}=0.04 D_{\|}$(Giacalone \& Jokipii 1999; Burger et al. 2000; Matthaeus et al. 2003).

A modeling technique for the particle transport and energy change in the spherical shock without perpendicular diffusion was described in detail by Kocharov et al. (2005). The particle energy change is caused by the divergence of the hydrodynamic velocity $\boldsymbol{u}=u(r, t) \boldsymbol{r} / r$. In the solar frame, plasma is assumed to be motionless until the shock arrives. The hydrodynamic velocity jumps at the shock according to the Rankine-Hugoniot jump condition and then linearly decreases to zero at $r=R_{\odot}$. The shock is described as the narrow continuous compression of a hyperbolic tangent form. The shock's speed, Alfvénic Mach number, and plasma beta, respectively, are $2 \times 10^{3} \mathrm{~km} \mathrm{~s}^{-1}, 5$, and 0.3 . Particle diffusion coefficients change in the shock front in the same way as the hydrodynamic speed does, from an upstream value, $D_{1 \mathrm{~A}(\mathrm{~B})}$, to a much smaller downstream value, $D_{2 \mathrm{~A}(\mathrm{~B})}=D_{1 \mathrm{~A}(\mathrm{~B})} / 20$ (Vainio \& Schlickeiser 1999). For the sake of simplicity, the upstream diffusion coefficient, $D_{1 \mathrm{~A}(\mathrm{~B})}$, is assumed to be independent of the distance from the Sun (cf. Laitinen \& Vainio 2003; Vainio et al. 2003). In tube A the upstream diffusion coefficient is $D_{\| 1 \mathrm{~A}}=2 \times 10^{7} \mathrm{~km}^{2} \mathrm{~s}^{-1}$ for the $0.1 \mathrm{MeV}$ proton. Both diffusion coefficients, $D_{\|}$and $D_{\perp}$, change in the direction perpendicular to the magnetic field from the corresponding value $D_{\mathrm{A}}$ in tube A to a much larger value in tube $\mathrm{B}: D_{\mathrm{B}}=50 D_{\mathrm{A}}$. The perpendicular dependence of diffusion coefficients, $D(Y)$, is adopted in hyperbolic tangent form: $\tanh \left[\left(Y-Y_{\mathrm{B}}\right) / \Delta\right]$ with the transition width $\Delta=0.1 R_{\odot}$.

The particle simulation box extends in $r$ from $R_{\odot}$ to $21 R_{\odot}$ and in $Y \equiv R_{o}(1-\cos \theta)$ from $Y(\theta=0)=0$ to $Y\left(\theta=\theta_{\mathrm{A}}\right)=R_{\odot}$. The outer boundary at $r=21 R_{\odot}$ is open, and our aim will be to find the energy spectrum and time profile of particles leaving the system here, mainly via the quiet tube B. All other boundaries are closed. At the boundary $\theta=0$, particles are bounced back to the simulation volume due to symmetry. The boundary condition at the box bottom, $r=R_{\odot}$, and in the middle of tube $\mathrm{A}, \theta=\theta_{\mathrm{A}}$, has a small effect on the spectrum of particles escaping at the top of tube B.

The shock acceleration from an upstream seed particle population starts upon the shock arrival at $r=1.5 R_{\odot}$ and continues until the end of the simulation period set at 30 minutes after the nominal shock launch at $r=R_{\odot}$. Seed particles are picked up upstream of the shock from the exponential energy distribution, $N_{\text {seed }}(E)=A_{1} \exp (-E / 0.3 \mathrm{MeV})$, with a constant volumetric density (Source 1 population). In addition, a source of unspecified nature, Source 2 , injects energetic protons behind the shock at the fixed distance from the Sun, $r=2 R_{\odot}$. Here, we inject into the system either monoenergetic, $10 \mathrm{MeV}$ protons, or a powerlaw spectrum of the $\geqslant 1 \mathrm{MeV}$ protons, $N_{\mathrm{SEP}}(E)=A_{2} E^{-2}$. Source 2 appears (opens) only after the shock has arrived at $2.5 R_{\odot}$ or $3.5 R_{\odot}$. Particles of each type, either shock accelerated from the sub-MeV seeds or released from the behind-theshock source, are separately registered at every Monte Carlo time step to accumulate the corresponding energy-coordinate distributions inside the simulation box. In addition, particles are registered upon their exit through the top of the simulation box to collect the energy spectra and time profiles of SEPs emitted into the interplanetary medium.

\section{RESULTS AND DISCUSSION}

We start with the instructive case of a short monoenergetic injection of Source 2 protons with no seed particles upstream of the shock. The monoenergetic, $10 \mathrm{MeV}$ protons are injected at $r=2 R_{\odot}$ only during the shock transit's time period from $r=2.5 R_{\odot}$ to $3, R_{\odot}$. Figure 3 shows the resulting time-volume-integrated energy spectrum of particles inside the simulation box and the time-integrated spectrum of escaping particles. The spectra can be split into three parts, $a, b$, and $c$, as shown in the figure. Protons are initially injected into the central energy layer $b$. A fraction of them overtakes the shock and escapes into the solar wind. However, some protons are decelerated and do not escape (part $a$ ), while others are significantly reaccelerated and then largely escape (part $c$ ). Spatial distribution of those three populations is shown in Figure 4. Shown are the maps of volumetric number density for the energy intervals $a-c$, registered in the shock frame and time integrated. In the shock frame, Source 2 drifts during its operational time from $-0.5 R_{\odot}$ to $-R_{\odot}$. The source location is 


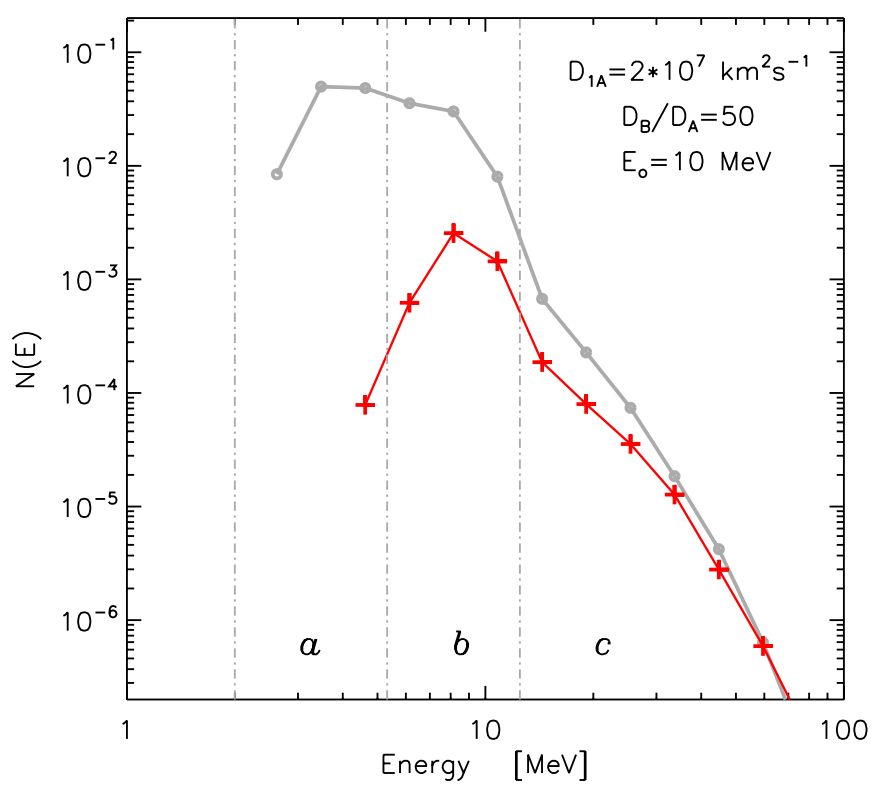

Figure 3. Time-integrated energy spectrum of the initially $10 \mathrm{MeV}$ protons of Source 2 after the reacceleration and adiabatic deceleration in the shocked plasma flow. Gray curve shows the volume averaged spectrum inside the simulation box. Red curve is for the escaping particles.

seen in the central energy layer (panel (b)). A confined proton population behind the shock in tube A suffers strong adiabatic deceleration and sinks to the lower energy layer (panel (a)). However, a significant fraction of protons from the $10 \mathrm{MeV}$ source overtakes the shock along the fast-transport tube B and then diffuses across the magnetic field lines upstream of the shock from tube B to the highly turbulent tube A where the shock acceleration is fast. Such particles are reaccelerated in the shock and thus lifted to the higher energy layer (panel (c)). Then the reaccelerated protons may return to tube B and escape along this tube to far upstream of the shock.

The simplest realistic model comprises two particle components: the uniformly distributed seed protons for the shock acceleration (Source 1) and the high-energy protons with a power-law spectrum $\left(\propto E^{-2}\right)$ released from the coronal source behind the shock (Source 2). The high-energy Source 2 located at $r=2 R_{\odot}$ starts its emission when the shock arrives at $r=3.5 R_{\odot}$ and continues with a constant rate until the end of the simulation period, $t_{\max }=30$ minutes. The modeling results are shown in Figure 5.

Panel (a) of Figure 5 shows the time-integrated spectrum of escaping shock-accelerated protons of Source 1 and the Source 2 protons escaping after the transmission through the shock. Also shown is the time-volume-integrated spectrum of the shockaccelerated protons inside the simulation box. This spectrum is dominated by particles near and downstream of the shock in tube A. The spectrum is somewhat softer than the standard steadystate/time-integrated spectrum in the planar shock (dashed line). The accelerated particle spectra in spherical shocks are known to be steeper than in planar shocks (e.g., Toptygin 1985). In the semi-transparent shock model, an additional steepening is caused by the energy-dependent escape of the accelerated particles through the perpendicular diffusion to tube B.

The modeling shows that SEPs from solar corona can penetrate through the interplanetary shock and appear in the particle energy spectrum above the high-energy rollover of particles
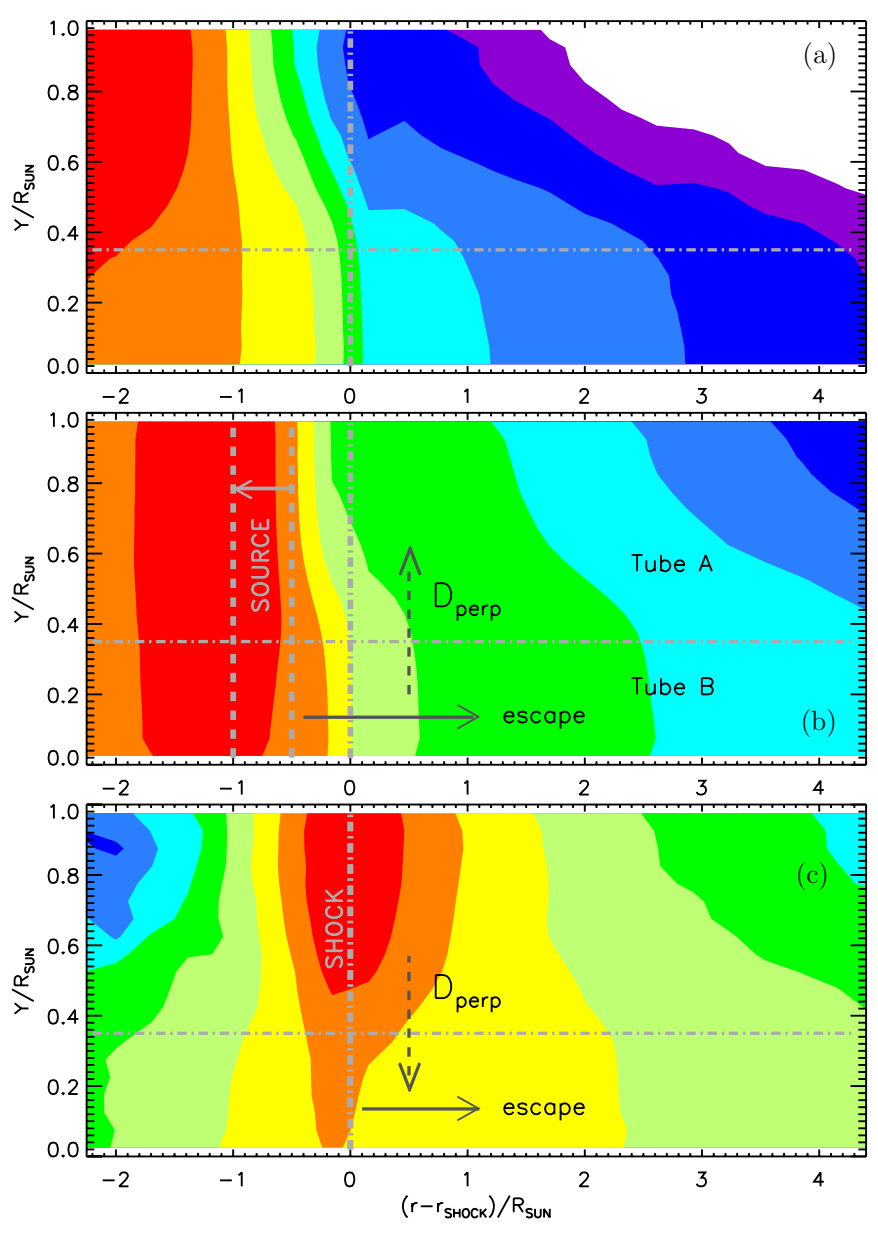

Figure 4. Map of the time-integrated proton distribution in three energy layers, (a) $2.3-5.3 \mathrm{MeV}$, (b) $5.3-12.5 \mathrm{MeV}$, and (c) $12.5-91 \mathrm{MeV}$, on the $r-Y$ plane in the shock frame for the mono-energetic Source $2\left(E_{o}=10 \mathrm{MeV}\right)$ that drifts between $r-r_{\mathrm{SHOCK}}=-0.5 R_{\odot}$ and $-R_{\odot}$. The energy intervals correspond to the intervals indicated in Figure 3 . Red shows the highest density of the accelerated particles (within the factor $e$ from the distribution maximum). Each following color level indicates one $e$-fold decline with respect to the previous one. The boundary between tubes $\mathrm{A}$ and $\mathrm{B}$ is shown at $Y=Y_{\mathrm{B}}+\Delta$. The integration time is 30 minutes.

accelerated by the shock. If the helium abundance of Source 2 differs from the seed particle abundance (Source 1), a step-like change in the helium-to-proton abundance ratio is expected at the transition to high energies. This is consistent with patterns observed in the first phase of the 1998 May 2 event (Figures 3(c) and (d) of Kocharov et al. 2007b). However, a difference in location and ion abundance of two SEP emissions does not necessarily mean that they were accelerated by different mechanisms. It is possible, for instance, that the high-energy component was previously accelerated by the same shock in a high coronal loop and later released from that loop after the magnetic reconnection behind the CME.

Figure 5(b) shows the model time profiles of the particle escape rate. Time profiles of protons accelerated by the shock on the open magnetic field lines (Source 1) are energy dependent, with an earlier rise of the lower energy channel in comparison to the higher energy one. This is indicative of the gradual gain of particle energy during the shock acceleration. Similar energydependent onset was observed in the time-shifted profiles of the $10-40 \mathrm{MeV}$ protons in the 1998 May 2 event (Kocharov et al. 2007b, Figure 3(a) therein). In contrast, the model profiles of protons transmitted from Source 2 rise simultaneously in 

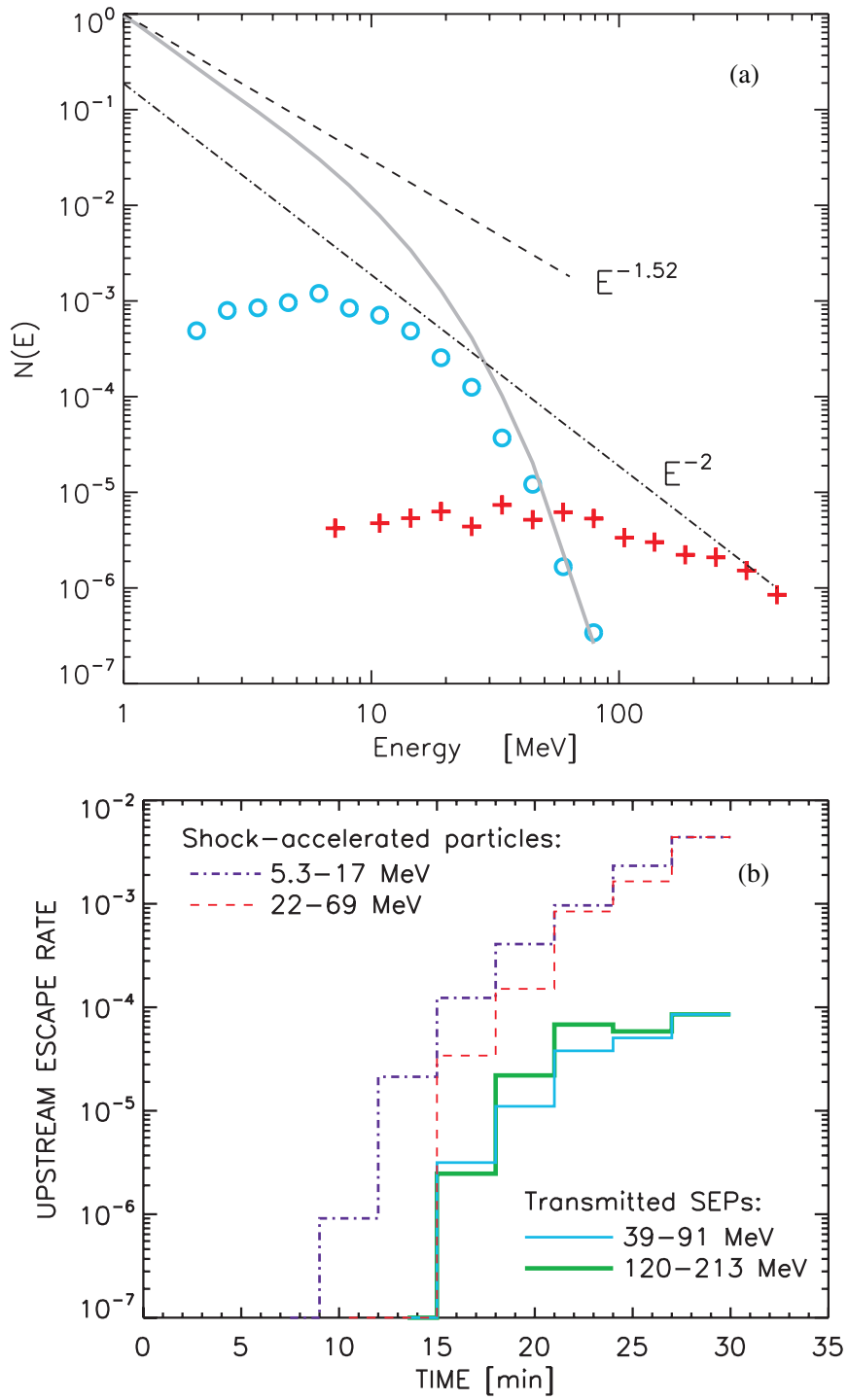

Figure 5. Energy spectrum and time profiles of shock-accelerated protons and protons transmitted through the shock from Source 2. (a) Time-volumeaveraged spectrum of protons accelerated by the shock from the upstream seed population (gray curve) and their spectrum emitted into the interplanetary medium (blue circles). Red plus signs are for the Source 2 protons that escape into the interplanetary space after traversing the shock. The integration time is 30 minutes. Source 2 injects into the system $2.5 \%$ of all particles with the spectrum shown with the dash-dotted line. The dashed line shows the steady state spectrum that would be expected in the planar shock of the same compression ratio: $N(E) \propto E^{-S}, S=(\sigma+0.5) /(\sigma-1)$ with $\sigma=3.86$. (b) Time profiles of escaping protons in two energy channels of shock-accelerated particles and two channels of particles transmitted from Source 2. Profiles of each pair are normalized to the same rate in the last time bin.

(A color version of this figure is available in the online journal.)

both energy channels. A similar, energy-independent rise was observed on 1998 May 2 in the time-shifted profiles of the high-energy proton channels, 50-140 MeV, and neutron monitors. Such simultaneous rise could be caused by the release of previously accelerated particles at the magnetic trap opening or by very fast acceleration.

\section{CONCLUSION}

We have modeled transmission of high-energy particles from the near-Sun source through the shock wave that accelerates SEPs between the Sun and the Earth. The transport channels, previously proposed for the prompt escape of the shockaccelerated particles, may also allow the high-energy particles from other sources, situated well behind the shock, to traverse the near-shock turbulent layer and to escape into the solar wind. The cross-field diffusion allows the different particle populations to appear at the same time and at the same location in the interplanetary space. The presence of the CME shock in solar wind between the Sun and the Earth does not necessarily prevent prompt access of high-energy particles from coronal sources to $1 \mathrm{AU}$, and equally the shock's transparency for the solar particles does not mean that the interplanetary shock does not accelerate SEPs.

This research was supported by the UK Science and Technology Facilities Council (grant ST/J001341/1) and by the Academy of Finland through projects 260596 and 272157 (ReSoLVE Center of Excellence).

\section{REFERENCES}

Axford, W. I., Leer, E., \& Skadron, G. 1977, in Proc. 15th ICRC (Plovdiv), 11, 132

Bell, A. R. 1978, MNRAS, 182, 147

Blandford, R. D., \& Ostriker, J. P. 1978, ApJL, 221, L29

Burger, R. A., Potgieter, M. S., \& Heber, B. 2000, JGR, 105, 27447

Cliver, E. W., \& Ling, A. G. 2007, ApJ, 658, 1349

Giacalone, J., \& Jokipii, J. R. 1999, ApJ, 520, 204

Gopalswamy, N., Xie, H., Yashiro, S., et al. 2012, SSRv, 171, 23

Kocharov, L., Laitinen, T., \& Vainio, R. 2013, ApJL, 778, L5

Kocharov, L., Lytova, M., Vainio, R., Laitinen, T., \& Torsti, J. 2005, ApJ, 620,1052

Kocharov, L., Saloniemi, O., Torsti, J., Kovaltsov, G., \& Riihonen, E. 2007a, ApJ, 654, 1121

Kocharov, L., Saloniemi, O., Torsti, J., et al. 2007b, ApJ, 659, 780

Kocharov, L., Vainio, R., Pomoell, J., et al. 2012, ApJ, 753, 87

Kocharov, L. G., Lee, J. W., Zirin, H., et al. 1994, SoPh, 155, 149

Kocharov, L. G., Torsti, J., Vainio, R., Kovaltsov, G. A., \& Usoskin, I. G. 1996, SoPh, 169, 181

Krymsky, G. F. 1977, DoSSR, 234, 1306

Laitinen, T., \& Vainio, R. 2003, AdSpR, 32, 2603

Lee, M. A. 2005, ApJS, 158, 38

Lee, M. A., \& Ryan, J. 1986, ApJ, 303, 829

Matthaeus, W. H., Qin, G., Bieber, J. W., \& Zank, G. P. 2003, ApJL, 590, L53

Ng, C. K., \& Reames, D. V. 2008, ApJL, 686, L123

Nitta, N. V., Liu, Y., DeRosa, M. L., \& Nightingale, R. W. 2012, SSRv, 171, 61 Toptygin, I. N. 1985, Cosmic Rays in Interplanetary Magnetic Fields (Geophys. Astrophys. Monogr. Vol. 27; Dordrecht: Reidel), 387

Torsti, J., Kocharov, L., Teittinen, M., \& Thompson, B. J. 1999, ApJ, 510, 460

Torsti, J., Riihonen, E., \& Kocharov, L. 2004, ApJL, 600, L83

Vainio, R., \& Laitinen, T. 2007, ApJ, 658, 622

Vainio, R., Laitinen, T., \& Fichtner, H. 2003, A\&A, 407, 713

Vainio, R., \& Schlickeiser, R. 1999, A\&A, 343, 303 